\title{
Residual Division Graph of Lattice Modules
}

\author{
Ganesh Gandal $D^{1},{ }^{1}$ R. Mary Jothi, ${ }^{1}$ and Narayan Phadatare ${ }^{2}$ \\ ${ }^{1}$ Department of Mathematics, Sathyabama Institute of Science and Technology, Chennai, India \\ ${ }^{2}$ Department of Mathematics, Savitribai Phule Pune University, Pune, India \\ Correspondence should be addressed to Ganesh Gandal; ganesh.gandal_skncoe@sinhgad.edu
}

Received 10 October 2021; Accepted 1 November 2021; Published 7 February 2022

Academic Editor: Francesca Tartarone

Copyright (C) 2022 Ganesh Gandal et al. This is an open access article distributed under the Creative Commons Attribution License, which permits unrestricted use, distribution, and reproduction in any medium, provided the original work is properly cited.

\begin{abstract}
Let $L$ be a multiplicative lattice and $M$ be a lattice module over $L$. In this paper, we assign a graph to $M$ called residual division graph $R G(M)$ in which the element $N \in M$ is a vertex if there exists $0_{M} \neq P \in M$ such that $N P=0_{M}$ and two vertices $N_{1}$, $N_{2}$ are adjacent if $N_{1} N_{2}=0_{M}$ (where $\left.N_{1} N_{2}=\left(N_{1}: I_{M}\right)\left(N_{2}: I_{M}\right) I_{M}\right)$. It is proved that such a graph with the greatest element $I_{M}$ which does not belong to the vertex set is nonempty if and only if $M$ is a prime lattice module. Also, we provide conditions such that $R G(M)$ is isomorphic to a subgraph of Zariski topology graph $G_{X}(M)$ with respect to $X$.
\end{abstract}

\section{Introduction}

In our everyday life, we found that numerous issues are dealt with the assistance of graphs. Extraordinarily, the idea of the coloring of graphs assumes a significant function in computer sciences. To examine the coloring of rings, I. Beck first presented the zero-divisor graphs of a commutative ring with unity (see [1]). This examination of the coloring of a commutative ring was further studied by Anderson and Naseer (see [2]).

The ring structure is firmly associated with ideals more than elements and so it has the right to present a graph with vertices as ideals instead of elements. In this direction, M. Behboodi et al. studied the annihilating-ideal graph $A G(R)$ with vertex set $V(A G(R))$ contain all those ideals of ring $R$ whose annihilators are nonzero(see $[3,4])$. Thereafter, Ansari-Toroghy et al. expanded this work for $R$-module $M$, where $R$ is a commutative ring. They studied the algebraic as well as topological properties of $M$ with the help of annihilating-submodule graph and the Zariski topology graph (see [5]). Recently, the idea of the zero divisor has likewise been applied in Boolean algebra, poset, and lattices (see $[6,7$, 78]).

A complete lattice $L=\left(L, \vee, \wedge, 0_{L}, 1_{L}\right)$ is a multiplicative if there is a defined binary operation called multiplication, denoted by ".”, which is commutative and associative such that the greatest element $1_{L}$ works as the multiplicative identity and for an arbitrary index set I $a .\left(\vee_{\alpha \in I} b_{\alpha}\right)=\vee_{\alpha \in I} a \cdot b_{\alpha}$, where $a, b_{\alpha \in L}$ and $0_{L}$ is the least element of $L$. It is interesting to note that the purpose behind the development of multiplicative lattice is to generalize lattices of ring ideals (see $[9,10])$. Also, it is observed that the annihilating-ideal graph of a commutative ring $R$ with unity has close ties with a multiplicative lattice of ideals of $R$. As far as the study of Johnson [11] is concerned, a lattice module is just an extension of a multiplicative lattice. It becomes worthy to study the graph $A G(R)$ over a commutative $\operatorname{ring} R$ with unity with the help of a lattice module $M$ over $L$.

Definition 1 (see [12]). A lattice module $M$ over the multiplicative lattice $L$ is a complete lattice with least element $0_{M}$ and greatest element $I_{M}$ if a multiplication between elements of $L$ and $M$, represented by $x N \in M$, where $x \in L$ and $N \in M$, which satisfies the following properties:
(1) $(x y) N=x(y N)$
(2) $\left(\mathrm{V}_{\alpha} x_{\alpha}\right)\left(\mathrm{V}_{\beta} N_{\beta}\right)=\left(\mathrm{V}_{\alpha \beta} x_{\alpha} N_{\beta}\right)$
(3) $1_{L} N=N$
(4) $0_{L} N=0_{M}$, for all $x, y, x_{\alpha} \in L$ and for all $N, N_{\beta} \in M$ 
Note that, for $P, Q \in M$ and $x \in L$, we define $(P: Q)=$ $\vee\{a \in L \mid a Q \leq P\}$ and $(P: x)=\vee\{K \in M \mid x K \leq P\}$. Here, the operation "I: " is called residual division (see [12]). Also, note that $A, C \in M$ with $A \leq C$; the interval $\{B \in M \mid A \leq B \leq C\}$ which is denoted by $C / A$ is a lattice module over a multiplicative lattice $L$ with the multiplication $a \cdot B=a B \vee A$, where $a \in L$ (see [12]).

Furthermore, for more definitions and concept of lattice modules and multiplicative lattice, see [9-19].

The semicomplement graph $\Gamma(M)$ of lattice module $M$ introduced and investigated by Phadatare et al. (see [20]). In the recent paper [5], Ansari-Toroghy and Habibi have highlighted the closed sets in Zariski topology on prime spectrum Spec $(M)$ of $R$-module $M$ and defined new graph called Zariski topology graph $G\left(\tau_{T}\right)$, where $T \subseteq \operatorname{Spec}(M)$ which is nonempty. They studied the relationship between $G\left(\tau_{T}\right)$ and annihilating-submodule graph $A G(M / \cap(T))$ (see [5]). Thereafter, this graph $G\left(\tau_{T}\right)$ generalized to lattice modules $M$ over a $C$-lattice $L$ and the Zariski topology graph $\mathrm{G}_{X}(M)$ was studied (see [21]).

Throughout the paper, $M$ denotes a lattice module over a multiplicative lattice $L$, and for $N, K \in M$, we define $N K=\left(N: I_{M}\right)\left(K: I_{M}\right) I_{M}$.

The aim of this paper is to generalize the annihilatingsubmodule graph of a module to the lattice module $M$ over a multiplicative lattice $L$ and introduce the residual division graph $R G(M)$ whose vertex set is $\{N \in M \mid$ there exists $0_{M} \neq K \in M$ such that $\left.N K=0_{M}\right\}$ and in $R G(M)$; two vertices $A, B$ are adjacent if and only if $A B=0_{M}$. Apart from this, we will investigate interrelationship between $G_{X}(M)$ and $R G(M / \wedge(X))$, where $\wedge(X)$ is the meet of all elements in $X$.

Throughout the paper, $M$ denotes a lattice module over a multiplicative lattice $L$.

\section{Some Graph Theoretic Notions}

We consider only undirected graphs. Thus, we adopt the notation $G=(V, E)$, where $V=V(G)$ is the set of vertices and $E=E(G)$ is the set of edges of $G$. A graph $G$ is an empty if $|V(G)|=\varnothing$. The number of edges incident on a vertex $v$ is called a degree of vertex $v$ and it is denoted by $d(v)$. A graph $G$ is said to be $k$-regular if the degree of each vertex in $G$ is $k$. Distance between the vertices $a$ and $b$ is the length of the shortest path between them, which is denoted by $d(a, b)$. Consider $d(a, b)=\infty$ if there is no path between $a$ and $b$. $\operatorname{diam}(G)=\sup \{d(a, c) \mid a, c \in V(G)\}$ is the diameter of a graph $G$. Length of shortest cycle in $G$ is called the girth of $G$, denoted by $\operatorname{gr}(G)$. A clique of graph $G$ is its maximal complete subgraph, and the minimum number of cliques required to cover all the vertices of graph $G$ is called the partition number, denoted by $\theta(G)$. In a graph $G$, a subset $S \subseteq V(G)$ is supposed to be independent if no two vertices in $S$ are adjacent. The size of maximum independent set in a graph $G$ called as independence number, denoted by $\alpha(G)$. For a vertex $x \in V(G), \Gamma_{G}(x)=\{y \mid x y \in E(G)\}$ denotes the set of all neighbors of $x^{\prime}$ in $G$. A graph $G$ is said to be perfect if $\theta(H)=\alpha(H)$, for every induced subgraph $H$ of $G$. Strongly perfect and very strongly perfect graphs are the classes of the perfect graph.
For further information, the reader may refer [22, 23].

\section{Residual Division Graph $R G(M)$}

Definition 2. The residual division graph $R G(M)$ of $M$ is a graph with vertices $V(R G(M))=\{N \in M \mid$ there exists $0_{M} \neq K \in M$ such that $\left.N K=0_{M}\right\}$, where distinct vertices $P$ and $Q$ are adjacent if and only if $P Q=0_{M}$.

Example 1. Figure 1 represents the residual division graph $R G(M)$ of $M$ with the vertex set $V(R G(M))=$ $\left\{0_{M}, A, B, C, Q\right\}$, where $M$ represents lattice module over $L$ (see Figure 2 and 3 ).

We essentially need the following two Lemmas throughout this article.

Lemma 1 (see [12]). For $x \in L$ and $P, Q, R \in M$, the following holds:

(1) If $P \leq Q$, then $(P: R) \leq(Q: R)$

(2) $(Q \wedge R: P)=(Q: P) \wedge(R: P)$

(3) $x \leq(x P: P)$

(4) $x(P: x) \leq P$

(5) $(P: Q) Q \leq P$

(6) If $P \leq Q$, then $(R: Q) \leq(R: P)$

Lemma 2 (see [12]). For $N \in M, \quad\left(\left(N: I_{M}\right) I_{M}: I_{M}\right)=$ $\left(N: I_{M}\right)$.

The following lemma gives a condition under which a nonzero proper element of lattice module $M$ is a vertex of $R G(M)$.

Lemma 3. Let $0_{M} \neq N$ be a proper element of $M$. Then, $N \in V$ $(R G(M))$ if $\left(0_{M}: N\right) \neq\left(0_{M}: I_{M}\right)$ or $\left(0_{M}:\left(N: I_{M}\right)\right) \neq 0_{M}$.

Proof. Suppose that $0_{M} \neq N$ is a proper element of $M$ and $\left(0_{M}: N\right) \neq\left(0_{M}: I_{M}\right)$. Then, $\left(0_{M}: N\right) I_{M} \neq 0_{M}$. Now, let $K=\left(0_{M}\right.$ : $N) I_{M}$. Therefore, $N K=\left(N: I_{M}\right)\left(\left(0_{M}: N\right) I_{M}: I_{M}\right) I_{M} \leq\left(N: I_{M}\right)$ $\left(0_{M}: N\right) I_{M}=\left(0_{M}: N\right)\left(N: I_{M}\right) I_{M} \leq\left(0_{M}: N\right) \quad N=0_{M}$. This implies that $N K=0_{M}$; consequently, $N \in V(R G(M))$.

Callialp and Tekir [14] introduced the notion of multiplication lattice modules.

Definition 3 (see [14]). A lattice module $M$ is said to be multiplication lattice module if for each $K \in M$ there exists an element $x \in L$ such that $K=x I_{M}$.

Lemma 4 (see [14]). A lattice module $M$ is a multiplication lattice module if and only if $K=\left(K: I_{M}\right) I_{M}$, for all $K \in M$.

Lemma 5. Let $0_{M} \neq N$ be a proper element of multiplication lattice module $M$. Then, $N \in V(R G(M))$ if and only if $\left(0_{M}:\left(N: I_{M}\right)\right) \neq 0_{M}$. 


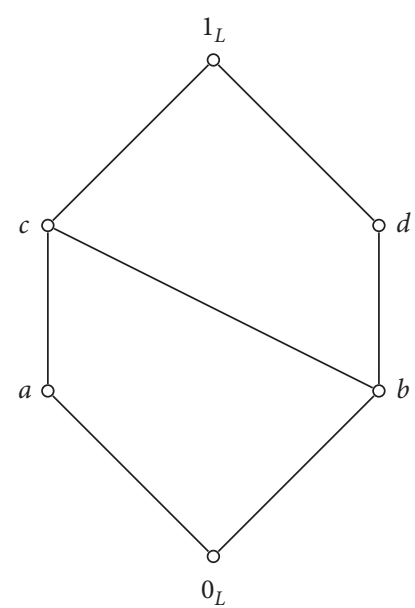

\begin{tabular}{|c|c|c|c|c|c|c|}
\hline$\cdot$ & $0_{L}$ & $\mathrm{a}$ & $\mathrm{b}$ & $\mathrm{c}$ & $\mathrm{d}$ & $1_{L}$ \\
\hline $0_{L}$ & $0_{L}$ & $0_{L}$ & $0_{L}$ & $0_{L}$ & $0_{L}$ & $0_{L}$ \\
\hline $\mathrm{a}$ & $0_{L}$ & $\mathrm{a}$ & $0_{L}$ & $\mathrm{a}$ & $0_{L}$ & $\mathrm{a}$ \\
\hline $\mathrm{b}$ & $0_{L}$ & $0_{L}$ & $0_{L}$ & $0_{L}$ & $\mathrm{~b}$ & $\mathrm{~b}$ \\
\hline $\mathrm{c}$ & $0_{L}$ & $\mathrm{a}$ & $0_{L}$ & $\mathrm{a}$ & $\mathrm{b}$ & $\mathrm{c}$ \\
\hline $\mathrm{d}$ & $0_{L}$ & $0_{L}$ & $\mathrm{~b}$ & $\mathrm{~b}$ & $\mathrm{~d}$ & $\mathrm{~d}$ \\
\hline $1_{L}$ & $0_{L}$ & $\mathrm{a}$ & $\mathrm{b}$ & $\mathrm{c}$ & $\mathrm{d}$ & $1_{L}$ \\
\hline
\end{tabular}

Figure 1: Multiplicative lattice $L$.

Proof. Suppose that $0_{M} \neq N \in M$ is a vertex of $R G(M)$. Then, by definition, there exists $0_{M} \neq K \in M$ such that $N K=0_{M}$. Therefore, $\left(N: I_{M}\right)\left(K: I_{M}\right) I_{M}=0_{M}$. Since $M$ is multiplication, by Lemma $4,\left(K: I_{M}\right) I_{M}=K$; therefore, $\left(N: I_{M}\right)\left(K: I_{M}\right) I_{M}=\left(N: I_{M}\right) K=0_{M}$. This implies $\left(0_{M}\right.$ : $\left.\left(N: I_{M}\right)\right)=K$. However, $K \neq 0_{M}$; therefore, $\left(0_{M}:(N\right.$ : $\left.\left.I_{M}\right)\right) \neq 0_{M}$. Converse part follows from Lemma 3.

Co-multiplication lattice module is introduced and characterized by F. Callialp et al. (see [16]).

Definition 4 (see [16]). Lattice module $M$ is called a comultiplication lattice module if for each $P \in M$, there exists an element $x \in L$ such that $P=\left(0_{M}: x\right)$.

The following characterization plays an important role in the study of residual division graph $R G(M)$.

Lemma 6 (see [16]). Lattice module $M$ is a co-multiplication if and only if $K=\left(0_{M}:\left(0_{M}: K\right)\right)$ for every element $K \in M$.

The following theorem is the immediate consequence of Lemma 6.

Theorem 1. Every proper element $0_{M} \neq N$ of a co-multiplication lattice module $M$ is a vertex of $R G(M)$.

Proof. Let $0_{M} \neq N$ is a proper element of co-multiplication lattice module $M$. By Lemma 3, to prove $N \in V(R G(M))$, we have to show that $\left(0_{M}: N\right) \neq\left(0_{M}: I_{M}\right)$ or $\left(0_{M}\right.$ : $\left.\left(N: I_{M}\right)\right) \neq 0_{M}$. Suppose that $\left(0_{M}: N\right)=\left(0_{M}: I_{M}\right)$. Then, by Lemma 1(6), $\left(0_{M}:\left(0_{M}: N\right)\right)=\left(0_{M}:\left(0_{M}: I_{M}\right)\right)$. Since $M$ is a co-multiplication lattice module over a $C$-lattice $L$, by

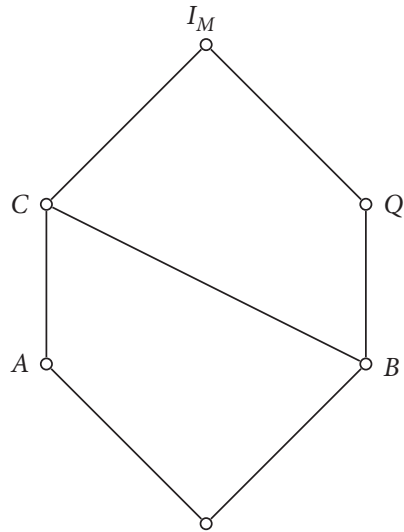

\begin{tabular}{|c|c|c|c|c|c|c|}
\hline$\cdot$ & $0_{M}$ & $\mathrm{~A}$ & $\mathrm{~B}$ & $\mathrm{C}$ & $\mathrm{Q}$ & $1_{M}$ \\
\hline 0 & $0_{M}$ & $0_{M}$ & $0_{M}$ & $0_{M}$ & $0_{M}$ & $0_{M}$ \\
\hline $\mathrm{a}$ & $0_{M}$ & $\mathrm{~A}$ & $0_{M}$ & $\mathrm{~A}$ & $0_{M}$ & $\mathrm{~A}$ \\
\hline $\mathrm{b}$ & $0_{M}$ & $0_{M}$ & $0_{M}$ & $0_{M}$ & $\mathrm{~B}$ & $\mathrm{~B}$ \\
\hline $\mathrm{c}$ & $0_{M}$ & $\mathrm{~A}$ & $0_{M}$ & $\mathrm{~A}$ & $\mathrm{~B}$ & $\mathrm{C}$ \\
\hline $\mathrm{d}$ & $0_{M}$ & $0_{M}$ & $\mathrm{~B}$ & $\mathrm{~B}$ & $\mathrm{Q}$ & $\mathrm{Q}$ \\
\hline $1_{L}$ & $0_{M}$ & $\mathrm{~A}$ & $\mathrm{~B}$ & $\mathrm{C}$ & $\mathrm{Q}$ & $1_{M}$ \\
\hline $\mathrm{:}$ & $0_{M}$ & $\mathrm{~A}$ & $\mathrm{~B}$ & $\mathrm{C}$ & $\mathrm{Q}$ & $1_{M}$ \\
\hline $0_{M}$ & $1_{L}$ & $\mathrm{~d}$ & $\mathrm{c}$ & $\mathrm{b}$ & $\mathrm{a}$ & $0_{L}$ \\
\hline $\mathrm{A}$ & $1_{L}$ & $1_{L}$ & $\mathrm{c}$ & $\mathrm{c}$ & $\mathrm{a}$ & $\mathrm{a}$ \\
\hline $\mathrm{B}$ & $1_{L}$ & $\mathrm{~d}$ & $1_{L}$ & $\mathrm{~d}$ & $\mathrm{c}$ & $\mathrm{b}$ \\
\hline $\mathrm{C}$ & $1_{L}$ & $1_{L}$ & $1_{L}$ & $\mathrm{c}$ & $\mathrm{c}$ & $\mathrm{c}$ \\
\hline $\mathrm{Q}$ & $1_{L}$ & $\mathrm{~d}$ & $1_{L}$ & $\mathrm{~d}$ & $1_{L}$ & $\mathrm{~d}$ \\
\hline $1_{M}$ & $1_{L}$ & $1_{L}$ & $1_{L}$ & $1_{L}$ & $1_{L}$ & $1_{L}$ \\
\hline
\end{tabular}

Figure 2: Lattice module $M$ over $L$.

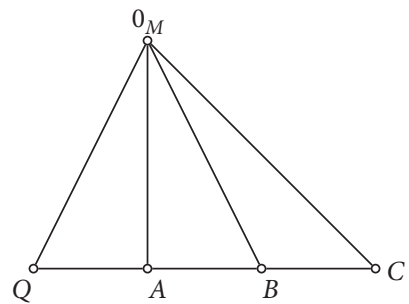

Figure 3: $R G(M)$.

Lemma $6, \quad N=\left(0_{M}:\left(0_{M}: N\right)\right)=\left(0_{M}:\left(0_{M}: I_{M}\right)\right)=I_{M}$ which is contradiction to $N<I_{M}$; consequently, $\left(0_{M}: N\right) \neq\left(0_{M}: I_{M}\right)$.

In the above three results, we studied various conditions on lattice module $M$ under which proper elements $0_{M} \neq N \in M$ becomes a vertex. However, then the natural question arises: can the greatest element $I_{M}$ be a vertex in residual division graph $R G(M)$ ?

The following lemma answers the above question.

Lemma 7. Greatest element $I_{M}$ of $M$ is a vertex if and only if there exists a proper element $0_{M} \neq N \in M$ such that $\left(N: I_{M}\right)=\left(0_{M}: I_{M}\right)$. 
Proof. Suppose that greatest element $I_{M}$ of a lattice module $M$ is a vertex. Then, there exists a $0_{M} \neq P \in M$ such that $P I_{M}=0_{M}$. Therefore, $\left(P: I_{M}\right)\left(I_{M}: I_{M}\right) I_{M}=0_{M}$. However, $\left(I_{M}: I_{M}\right) I_{M}=I_{M}$; therefore, $\left(P: I_{M}\right) I_{M}=0_{M}$, and hence, $\left(P: I_{M}\right)=\left(0_{M}: I_{M}\right)$. Conversely, suppose that $\left(N: I_{M}\right)=$ $\left(0_{M}: I_{M}\right)$, where $N$ is a proper element of $M$ with $0_{M} \neq N$. Then, $N I_{M}=\left(N: I_{M}\right)\left(I_{M}: I_{M}\right) I_{M}=\left(N: I_{M}\right) I_{M}$ because of $\left(I_{M}: I_{M}\right) I_{M}=I_{M}$. Since $\left(N: I_{M}\right)=\left(0_{M}: I_{M}\right)$, we have $N I_{M}=\left(N: I_{M}\right) I_{M}=\left(0_{M}: I_{M}\right) I_{M} \leq 0_{M}$. By Lemma 1(5), $\left(0_{M}: I_{M}\right) I_{M} \leq 0_{M}$; therefore, $N I_{M}=0_{M}$; hence, the greatest element $I_{M}$ is a vertex.

Theorem 2. Let the greatest element $I_{M}$ of $M$ is not a vertex of $R G(M)$. Then, $0_{M} \neq N \in M$ is a vertex if and only if $\left(0_{M}:\left(N: I_{M}\right)\right) \neq 0_{M}$.

Proof. Suppose that $0_{M} \neq N \in M$ is in $V(R G(M))$. Then, there exists $0_{M} \neq P \in M$ such that $N P=0_{M}$. Therefore, $N P=\left(N: I_{M}\right)\left(P: I_{M}\right) I_{M}=0_{M}$ and so $\left(P: I_{M}\right) I_{M} \leq$ $\left(0_{M}:\left(N: I_{M}\right)\right)$. Since $I_{M}$ is not a vertex, by Lemma 7 , $\left(P: I_{M}\right) I_{M} \neq 0_{M}$, and hence, $\left(0_{M}:\left(N: I_{M}\right)\right) \neq 0_{M}$. Converse follows from Lemma 3.

In [13], Al-Khouja studied the relationship between the maximal (prime) elements of lattice module $M$ and the maximal (prime) elements of multiplicative lattice $L$. "If $N$ is a prime element of a lattice module $M$ over a multiplicative lattice $L$, then $\left(N: I_{M}\right)$ is a prime element of multiplicative lattice $L$ (see [13])."

According to Callialp et al. (see [15]), a lattice module $M$ over a multiplicative lattice $L$ is prime if the least element $0_{M}$ is prime element of $M$.

The following characterization is done by Callialp et al. (see [15]).

Lemma 8 (see [15]). Least element $0_{M}$ of $M$ is a prime if and only if $\left(0_{M}: I_{M}\right)=\left(0_{M}: N\right)$, for all $0_{M} \neq N \in M$.

Lemma 8 helps us to characterize the prime lattice module.

Theorem 3. Let the greatest element $I_{M}$ of $M$ is not a vertex of $R G(M)$. Then, $R G(M)=\varnothing$ if and only if $M$ is a prime lattice module.

Proof. Suppose that $R G(M)=\varnothing$ and $M$ is not a prime lattice module over $L$. Then, least element $0_{M}$ is not a prime element of $M$. Therefore, by Lemma 8, there exists proper element $0_{M} \neq N \in M$ such that $\left(0_{M}: I_{M}\right) \neq\left(0_{M}: N\right)$, and hence, by Lemma $3, N$ is in $V(R G(M))$, contradiction to $R G(M)=\varnothing$; consequently, $M$ is prime. Conversely, $M$ is a prime, and there exists $N \in M$ such that $N \in V(R G(M))$. Then, by definition, there exists $0_{M} \neq K$ in $M$ such that $N K=0_{M}$. This implies that $\left(N: I_{M}\right)\left(K: I_{M}\right) I_{M}=0_{M}$, so $\left(N: I_{M}\right)\left(K: I_{M}\right)=\left(0_{M}: I_{M}\right)$. Since $0_{M}$ is a prime element of $M,\left(0_{M}: I_{M}\right)$ prime element of $L$. Therefore, $\left(N: I_{M}\right)=$ $\left(0_{M}: I_{M}\right)$ or $\left(K: I_{M}\right)=\left(0_{M}: I_{M}\right)$. This follows by Lemma 7 that $I_{M}$ is a vertex, a contradiction. Consequently, $R G(M)=\varnothing$.
Theorem 4. For given $M, R G(M)$ is connected and $\operatorname{diam}(R G(M)) \leq 3$.

Proof. Suppose that $P, Q \in V(R G(M))$ such that $P \neq Q$. If $P Q=0_{M}$, by definition, we have a path $P-Q$ of length one. Now, suppose that $P Q \neq 0_{M}$.

Case (1): if $P^{2}=P P=0_{M}$ and $Q^{2}=Q Q=0_{M}$, then $P P Q=\left(P: I_{M}\right)\left(P: I_{M}\right)\left(Q: I_{M}\right) I_{M}=0_{M}$ and $P Q Q=$ $\left(P: I_{M}\right)\left(Q: I_{M}\right)\left(Q: I_{M}\right) I_{M}=0_{M}$. This implies that $P$ is adjacent to $P Q$ and $P Q$ is adjacent to $Q$, i.e., $P-P Q-Q$ is a path of length equal to 2 .

Case (2): if $P^{2}=0_{M}$ and $Q^{2} \neq 0_{M}$, since $Q \in V(R G(M))$, there exists $0_{M} \neq Q_{1} \in M$ such that $Q Q_{1}=0_{M}$. If $P Q_{1}=0_{M}$, then we have a path $P-Q_{1}-$ $Q$ with $d(P, Q)=2$. Suppose that $P Q_{1} \neq 0_{M}$. Then, $P P Q_{1}=P^{2} Q_{1}=0_{M}$, and therefore, $P-P Q_{1}-Q$ is a path of length 2 because $Q Q_{1}=0_{M}$. Similarly, if $Q^{2}=$ $0_{M}$ and $P^{2} \neq 0_{M}$, then we have a path with length 2 .

Case (3): if $P Q \neq 0_{M}, P^{2} \neq 0_{M}$, and $Q^{2} \neq 0_{M}$, by definition, there exist $0_{M} \neq N, K$ such that $P N=0_{M}=Q K$. If $N=K$, then $P-N=K-Q$ is a path of length 2 . Now, suppose that $N \neq K$ and $N K=0_{M}$. Since $P N=0_{M}=Q K$, we have a path $P-N-K-Q$ of length 3, i.e., $d(P, Q)=3$. Above cases implies that $d(P, Q) \leq 3$; consequently, $\operatorname{diam}(R G(M)) \leq 3$.

Corollary 1. If $R G(M)$ contain a cycle, then $\operatorname{gr}(R G(M)) \leq 4$.

In [21], Phadatare et al. introduced the quasi-prime element of $M$.

Definition 5 (see [21]). A proper element $N \in M$ is said to be quasi-prime if $\left(N: I_{M}\right)$ is a quasi-prime element of $L$.

The following lemma follows from Theorem 3.

Lemma 9. Let the greatest element $I_{M}$ of $M$ is not a vertex of $R G(M)$. If $R G(M)=\varnothing$, then the least element $0_{M}$ is a quasiprime element of $M$.

$\operatorname{Spec}^{q}(M)$ is a collection of all quasi-prime elements of $M$ and for $N \in M$, set $D(N)=\left\{K \in \operatorname{Spec}^{q}(M) \mid\left(N: I_{M}\right) \leq(K\right.$ : $\left.\left.I_{M}\right)\right\}$ (see [21]).

We basically need the following lemma.

Lemma 10 (see [21]). Let $M$ be a lattice module and $Y \subseteq$ $\operatorname{Spec}^{q}(M)$. Then, $Y \subseteq D(N)$ if and only if $\left(N: I_{M}\right) \leq(\wedge$ $\left.(Y): I_{M}\right)$.

Phadatare et al. [21] employed closed set $D(N)$ to introduce the Zariski topology graph $G_{X}(M)$ with respect to $X \subseteq \operatorname{Spec}^{q}(M)$.

Definition 6. For $X \subseteq \operatorname{Spec}^{q}(M)$, we define an undirected graph $G_{X}(M)$ associated with $X$, called Zasiski topology graph with respect to $X$ with vertex set $V\left(G_{X}(M)\right)=\{N \in M \mid$ there exists $0_{M} \neq K \in M$ such that $D(N) \cup D(K)=X$ and $D$ 
$(N) \neq X, D(K) \neq X\}$ and distinct vertices $N$ and $K$ are adjacent if and only if $D(N) \cup D(K)=X$.

Remark 1. By Proposition 3.1 of [21] and Lemma 2, for $N$, $K \in M, D(N K)=D\left(\left(N: I_{M}\right)\left(K: I_{M}\right) I_{M}\right)=D(N) \cup D(K)$.

Note that, for $Y \subseteq \operatorname{Spec}^{q}(M)$, an interval $\{K \in M: \wedge$ $(Y) \leq K \leq M\}$ denoted by $M / \wedge(Y)=\bar{M}$ is a lattice module over a multiplicative lattice $L$ with the multiplication $a \cdot K=a K \vee \wedge(Y)$, where $a \in L$.

Theorem 5. Let $M$ be a lattice module, and $M / \wedge(Y)=\overline{I_{M}}$ is not a vertex of $R G(M / \wedge(Y))$. Then, $R G(M / \wedge(Y))$ is isomorphic to subgraph of $G_{Y}(M)$.

Proof. Suppose that $\bar{N}=N / \wedge(Y) \in V(R G(M / \wedge(Y)))$. By definition, there exists $\wedge(Y) \neq K / \wedge(Y) \in M / \wedge(Y)$ such that $N /$ $\wedge(Y)$ is adjacent to $K / \wedge(Y)$. Therefore, $\overline{N K}=N / \wedge(Y) K /$ $\wedge(Y)=\left(N / \wedge(Y): I_{M} / \wedge(Y)\right)\left(K / \wedge(Y): I_{M} / \wedge(Y)\right) I_{M} / \wedge(Y)$ $=\wedge(Y)$. Note that $\left(N / \wedge(Y): I_{M} / \wedge(Y)\right) \quad\left(K / \wedge(Y): I_{M} / \wedge\right.$ $(Y))=\left(\left(N: I_{M}\right) \quad\left(K: I_{M}\right) I_{M} \vee \wedge(Y)\right) / \wedge(Y)$. Therefore, we have $\overline{N K}=N / \wedge(Y) K / \wedge(Y)=\left(\left(N: I_{M}\right)\left(K: I_{M}\right) I_{M} \vee \wedge(Y)\right) /$ $\wedge(Y)=\wedge(Y)$. This impies that $N K=\left(N: I_{M}\right)\left(K: I_{M}\right) I_{M}$ $\leq \wedge(Y))$; therefore, by Lemma $1(1),\left(N K: I_{M}\right) \leq\left(\wedge(Y): I_{M}\right)$, and hence, by Lemma $10, D(N K)=Y$. Since $D(N K)$ $=D(N) \cup D(K)$, we have $Y=D(N) \cup D(K)$. If $D(N)=Y$, then $\left(N: I_{M}\right)=\left(\wedge(X): I_{M}\right)$; therefore, by Lemma $7, \overline{I_{M}}$ is a vertex of $R G(M / \wedge(Y))$ which is a contradiction; consequently, $D(N) \neq Y$. In the same line, we have $D(K) \neq Y$. Thus, $N \in V\left(G_{Y}(M)\right)$ such that $N$ is adjacent to $K$.

Corollary 2 (see [21]). For $X \subseteq \operatorname{Spec}^{q}(M), G_{X}(M)$ is nonempty if and only $X=D(\wedge(X))$ and $\wedge(X) \notin \operatorname{Spec}^{q}(M)$.

The following is the characterization of nonempty residual division graph $R G(M / \wedge(Y))$.

Theorem 6. If $M / \wedge(Y)=\overline{I_{M}}$ is not a vertex of $R G(M / \wedge(Y))$, then $R G(M / \wedge(Y))=\varnothing$ if and only if $G_{Y}(M)=\varnothing$.

Proof. Suppose that $\overline{I_{M}}$ is not a vertex of $R G(M / \wedge(Y))$ and $R G(M / \wedge(Y))=\varnothing$. Then, by Lemma $9, \wedge(Y)$ is a quasiprime element of $M$; therefore, by Corollary $2, G_{Y}(M)=\varnothing$. Converse follows from Theorem 6 .

Theorem 7. The greatest element $M / \wedge(Y)=\overline{I_{M}}$ is a vertex in $R G(M / \wedge(Y))$ if there exists $N<I_{M}$ such that $\wedge(Y)<N$ and $D(N)=Y$.

Proof. Suppose there exists $N<I_{M}$ such that $\wedge(Y)<N$ and $D(N)=Y$. To prove that $\overline{I_{M}} \in V(R G(M / \wedge(Y)))$, it suffices to prove that there exists $\wedge(Y) \neq \bar{K}$ such that $\overline{K I_{M}}=\wedge(Y)$. Note that $\wedge(Y)<N<I_{M}$. By definition, $\overline{N I_{M}}=$ $N / \wedge(Y) I_{M} / \wedge(Y)=\left(N / \wedge(Y): I_{M} / \wedge(Y)\right)\left(I_{M} / \wedge(Y): \quad I_{M} / \wedge\right.$ $(Y)) I_{M} / \wedge(Y)=\left(N: I_{M}\right) I_{M} \vee \wedge(Y) / \wedge(Y)$. Since $D(N)=Y$, we have, for all $P \in Y,\left(N: I_{M}\right) \leq\left(P: I_{M}\right)$; therefore, $\left(N: I_{M}\right) \leq \wedge_{P \in Y}\left(P: I_{M}\right)$, and hence, by Lemma $1(2)$, $\left(N: I_{M}\right) \leq\left(\wedge_{P \in Y} P: I_{M}\right)$. This implies that $\left(N: I_{M}\right) \leq$
$\left(\wedge(Y): I_{M}\right)$, i.e., $\left(N: I_{M}\right) I_{M} \leq \wedge(Y)$; therefore, $\left(N: I_{M}\right) I_{M}$ $\vee \wedge(Y) / \wedge(Y)=\wedge(Y)$; consequently, $\overline{I_{M}}$ is a vertex in $R G(M / \wedge(Y))$.

For $N<I_{M}, \sqrt{N}$ represents q-radical of $N$, and it is defined as $\sqrt{N}=\wedge D(N)$. If $\sqrt{N}=N$, then $N$ is said to be $q$-radical element of $M$.

"If the natural map $\psi: \operatorname{Spec}^{q}(M) \longrightarrow \operatorname{Spec}^{q}$ $\left(L /\left(0_{M}: I_{M}\right)\right)$ defined by $\psi(N)=\overline{\left(N: I_{M}\right)}$ surjective, then $\sqrt{\left(N: I_{M}\right)}=\left(\sqrt{N}: I_{M}\right)($ see $[24]) . "$

Theorem 8. If the natural map $\psi$ is surjective and $A, B$ are adjacent vertices in $G_{Y}(M)$. Then, $\sqrt{A} / \wedge(Y)$ and $\sqrt{B} / \wedge(Y)$ are adjacent in $R G(M / \wedge(Y))$.

Proof. To show that $\sqrt{A} / \wedge(Y)$ and $\sqrt{B} / \wedge(Y)$ are adjacent in $R G(M / \wedge(Y))$, we have to prove that $\sqrt{\left(A: I_{M}\right) I_{M}} / \wedge(Y) \neq \wedge$ $(Y)$ and $\sqrt{\left(B: I_{M}\right) I_{M}} / \wedge(Y) \neq \wedge(Y)$ with $\sqrt{\left(A: I_{M}\right) I_{M}} / \wedge(Y)$ $\sqrt{\left(B: I_{M}\right) I_{M}} / \wedge(Y)=\wedge(Y)$. Suppose that $A, B$ are adjacent vertices in $G_{Y}(M)$. Then, we have $D(A) \cup D(B)=Y$ with $D$ $(A), D(B) \neq Y$. However, $D(A) \cup D(B)=D\left(\left(A: I_{M}\right)\left(B: I_{M}\right)\right.$ $\left.I_{M}\right)$; therefore, $Y=D\left(\left(A: I_{M}\right)\left(B: I_{M}\right) I_{M}\right)$, and hence, $\wedge(Y)=$ $\wedge D\left(\left(A: I_{M}\right)\left(B: I_{M}\right) I_{M}\right)=\sqrt{\left(A: I_{M}\right)\left(B: I_{M}\right) I_{M}} \leq \sqrt{\left(A: I_{M}\right) I_{M}}$ $\cap \sqrt{\left(B: I_{M}\right) I_{M}}$. Thus, $\wedge(Y) \leq \sqrt{\left(A: I_{M}\right) I_{M}} \cap \sqrt{\left(B: I_{M}\right) I_{M}}$. Also, note that $\sqrt{\left(A: I_{M}\right) I_{M}} / \wedge(Y) \quad \sqrt{\left(B: I_{M}\right)} \quad I_{M} / \wedge(Y)=$ $\left(\sqrt{\left(A: I_{M}\right) I_{M}} / \wedge(Y): I_{M} / \wedge(Y)\right)\left(\sqrt{\left(B: I_{M}\right) I_{M}} / \wedge(Y): I_{M} / \wedge\right.$ $(Y)) I_{M} / \wedge(Y)=\left(\left(\sqrt{\left(A: I_{M}\right) I_{M}}: I_{M}\right)\left(\sqrt{\left(B: I_{M}\right) I_{M}}: I_{M}\right)\right) I_{M}$ $\vee \wedge(Y) / \wedge(Y)$. Since $\psi$ is surjective and $D(N)=D\left(\left(N: I_{M}\right)\right.$ $\left.I_{M}\right)$, we have $\left(\sqrt{\left(A: I_{M}\right) I_{M}}: I_{M}\right)=\left(\sqrt{A}: I_{M}\right)=\sqrt{\left(A: I_{M}\right)}$; therefore, $\sqrt{\left(A: I_{M}\right) I_{M}} / \wedge(Y) \sqrt{\left(B: I_{M}\right) I_{M}} / \wedge(Y)=((\sqrt{(A:}$ $\left.\left.\left.I_{M}\right) I_{M}: I_{M}\right)\left(\sqrt{\left(B: I_{M}\right) I_{M}}: I_{M}\right)\right) I_{M} \vee \wedge(Y) / \wedge(Y)=\sqrt{(A:}$ $\left.I_{M}\right) \sqrt{\left(B: I_{M}\right)} I_{M} \vee I_{M} / \wedge(Y)$. However, $\sqrt{\left(A: I_{M}\right)} \sqrt{\left(B: I_{M}\right)}$ $I_{M} \leq \sqrt{\left(A: I_{M}\right)\left(B: I_{M}\right)} I_{M} \leq \sqrt{\left(A: I_{M}\right)\left(B: I_{M}\right) I_{M}}=\sqrt{A B}=$ $\wedge Y)$; therefore, $\sqrt{\left(A: I_{M}\right) I_{M}} / \wedge(Y) \quad \sqrt{\left(B: I_{M}\right) I_{M}} / \wedge(Y)=$ $\sqrt{\left(A: I_{M}\right)} \sqrt{\left(B: I_{M}\right)} I_{M} \vee I_{M} / \wedge(Y)=\wedge(Y)$. Now, it remains to prove that $\sqrt{\left(A: I_{M}\right) I_{M}} / \wedge(Y) \neq \wedge(Y)$ and $\sqrt{\left(A: I_{M}\right) I_{M}} / \wedge(Y)$ $\neq \wedge(Y)$. If $\sqrt{\left(A: I_{M}\right) I_{M}} / \wedge(Y)=\wedge(Y)$, then $\sqrt{\left(A: I_{M}\right) I_{M}}=$ $\wedge(Y)$. Therefore, $\left(A: I_{M}\right) I_{M} \leq \sqrt{\left(A: I_{M}\right) I_{M}}=\wedge(Y)$, and hence, $\left(A: I_{M}\right) \leq\left(\wedge(Y): I_{M}\right)$. By Lemma $10, D(N)=Y$, a contradiction. Consequently, $\sqrt{\left(A: I_{M}\right) I_{M}} / \wedge(Y) \neq \wedge(Y)$. Similarly, we have $\sqrt{\left(B: I_{M}\right) I_{M}} / \wedge(Y) \neq \wedge(Y)$.

\section{Conclusion}

In this paper, we introduced the residual division graph of the lattice module and characterized the co-multiplication lattice module and prime lattice module. Also, we characterized vertex set of residual division graph of the multiplication lattice module. We found that the residual division graph of an interval lattice module is isomorphic with the Zariski topology graph.

\section{Data Availability}

The data from previous studies were used to support this study. They are cited at relevant places within the article as references. 


\section{Conflicts of Interest}

The authors declare that they have no conflicts of interest.

\section{References}

[1] I. Beck, "Coloring of commutative rings," Journal of Algebra, vol. 116, no. 1, pp. 208-226, 1988.

[2] D. D. Anderson and M. Naseer, "Beck's coloring of a commutative ring," Journal of Algebra, vol. 159, no. 2, pp. 500-514, 1993.

[3] M. Behboodi and Z. Rakeei, "The annihilating-ideal graph of commutative rings-I," Journal of Algebra and Its Applications, vol. 10, no. 4, pp. 727-739, 2011.

[4] M. Behboodi and Z. Rakeei Z, "The annihilating-ideal graph of commutative rings-II," Journal of Algebra and Its Applications, vol. 10, no. 4, pp. 741-753, 2011.

[5] H. Ansari-Toroghy and S. Habibi, "The zariski topology-graph of modules over commutative rings," Communications in Algebra, vol. 42, no. 8, pp. 3283-3296, 2014.

[6] D. F. Anderson, R. Levy, and J. Shapiro, "Zero-divisor graphs, von Neumann regular rings, and Boolean algebras," Journal of Pure and Applied Algebra, vol. 180, no. 3, pp. 221-241, 2003.

[7] E. Estaji and K. Khashyarmanesh, "The zero-divisor graph of a lattice," Results in Mathematics, vol. 61, no. 1-2, pp. 1-11, 2012.

[8] R. Halaš and M. Jukl, “On Beck's coloring of posets," Discrete Mathematics, vol. 309, pp. 4584-4589, 2009.

[9] R. P. Dilworth, "Abstract commutative ideal theory," Pacific Journal of Mathematics, vol. 12, no. 2, pp. 481-498, 1962.

[10] M. Ward and R. P. Dilworth, "Residuated lattices," Transactions of the American Mathematical Society, vol. 45, no. 3, pp. 335-354, 1939.

[11] E. W. Johnson, A-Transforms of Noether Lattices, Ph.D. Dissertation, University of California, Los Angeles, CA, USA, 1966.

[12] J. A. Johnson, "a-adic completions of Noetherian lattice modules," Fundamenta Mathematicae, vol. 66, pp. 341-371, 1970.

[13] E. A. AL-Khouja, "Maximal elements and prime elements in lattice modules," Damascus University Journal of Basic Sciences, vol. 19, pp. 9-20, 2003.

[14] F. Callialp and U. Tekir, "Multiplication lattice modules, Iran," Iranian Journal of Science and Technology, Transaction A, Science, vol. 4, pp. 309-313, 2011.

[15] F. Callialp, U. Tekir, and E. AslanKarayigit, "On multiplication lattice modules," Hacettepe Journal of Mathematics and Statistics, vol. 43, no. 4, pp. 571-579, 2014.

[16] F. Callialp, U. Tekir, and G. Ulucak, "Comultiplication lattice modules, Iran," Iranian Journal of Science and Technology, Transaction, vol. A2, no. 39, pp. 213-220, 2015.

[17] R. H. Lee, "Covers and associated primes in noetherian lattice modules," Houston Journal of Mathematics, vol. 5, no. 1, pp. 219-239, 1979.

[18] N. K. Thakare and C. S. Manjarekar, "Abstract spectral theory: multiplicative lattices in which every character is contained in a unique maximal character," in Algebra and its Applications, pp. 265-276, Marcel Dekker, New York, USA, 1984.

[19] N. K. Thakare, C. S. Manjarekar, and S. Maeda, "Abstract spectral theory II: minimal characters and minimal spectrums of multiplicative lattices," Acta Scientiarum Mathematicarum, vol. 52, pp. 53-67, 1988.

[20] N. Phadatare, V. Kharat, and S. Ballal, "Semi-complement graph of lattice modules," Soft Computing, vol. 23, no. 12, pp. 3973-3978, 2019.
[21] N. Phadatare, V. Kharat, and S. Ballal, Zariski Topology Graph of Quasi-Prime Spectrum of Lattice Modules.

[22] F. Harary, Graph Theory, Narosa, New Delhi, India, 1988.

[23] D. B. West, Introduction to Graph Theory, Prentice-Hall of India, New Delhi, India, 2nd edition, 2002.

[24] N. Phadatare, S. Ballal, and V. Kharat, "On the maximal spectrum of lattice modules," Southeast Asian Bulletin of Mathematics, vol. 44, pp. 105-117, 2020. 